This is an author produced version of a paper published in Biosensors and Bioelectronics. This paper has been peer-reviewed but does not include the final publisher proof-corrections or journal pagination.

Citation for the published paper:

Nocchi, Sarah; Björklund, Sebastian; Svensson, Birgitta; Engblom, Johan; Ruzgas, Tautgirdas. (2017). Electrochemical monitoring of native catalase activity in skin using skin covered oxygen electrode. Biosensors and Bioelectronics, vol. 93, p. null

URL: https://doi.org/10.1016/j.bios.2017.01.001

Publisher: Elsevier

This document has been downloaded from MUEP (https://muep.mah.se) / DIVA (https://mau.diva-portal.org). 


\title{
Electrochemical monitoring of native catalase activity in skin using skin covered oxygen electrode
}

Sarah Nocchi ${ }^{1,2}$, Sebastian Björklund ${ }^{1,2}$, Birgitta Svensson ${ }^{3}$, Johan Engblom ${ }^{1,2}$, and

Tautgirdas Ruzgas ${ }^{1,2^{*}}$

\author{
${ }^{1}$ Department of Biomedical Sciences, Faculty of Health and Society, Malmö University, SE-205 06, \\ Malmö, Sweden. \\ ${ }^{2}$ Biofilms - Research Center for Biointerfaces, Malmö University, SE-205 06, Malmö, Sweden \\ ${ }^{3}$ Bioglan AB, SE-202 13 Malmö, Sweden \\ *corresponding author: tautgirdas.ruzgas@mah.se
}

\section{Abstract}

A skin covered oxygen electrode, SCOE, was constructed with the aim to study the enzyme catalase, which is part of the biological antioxidative system present in skin. The electrode was exposed to different concentrations of $\mathrm{H}_{2} \mathrm{O}_{2}$ and the amperometric current response was recorded. The observed current is due to $\mathrm{H}_{2} \mathrm{O}_{2}$ penetration through the outermost skin barrier (referred to as the stratum corneum, $\mathrm{SC}$ ) and subsequent catalytic generation of $\mathrm{O}_{2}$ by catalase present in the underlying viable epidermis and dermis. By tape-stripping the outermost skin layers we demonstrate that $\mathrm{SC}$ is a considerable diffusion barrier for $\mathrm{H}_{2} \mathrm{O}_{2}$ penetration. Our experiments also indicate that skin contains a substantial amount of catalase, which is sufficient to detoxify $\mathrm{H}_{2} \mathrm{O}_{2}$ that reaches the viable epidermis after exposure of skin to high concentrations of peroxide (0.5-1 $\left.\mathrm{mM} \mathrm{H}_{2} \mathrm{O}_{2}\right)$. Further, we demonstrate that the catalase activity is reduced at acidic $\mathrm{pH}$, as compared with the activity at $\mathrm{pH}$ 7.4. Finally, experiments with often used penetration enhancer thymol shows that this compound interferes with the catalase reaction. Health aspect of this is briefly discussed. Summarizing, the results of this work show that the SCOE can be utilized to study a broad spectrum of issues involving the function of skin catalase in particular, and the native biological antioxidative system in skin in general.
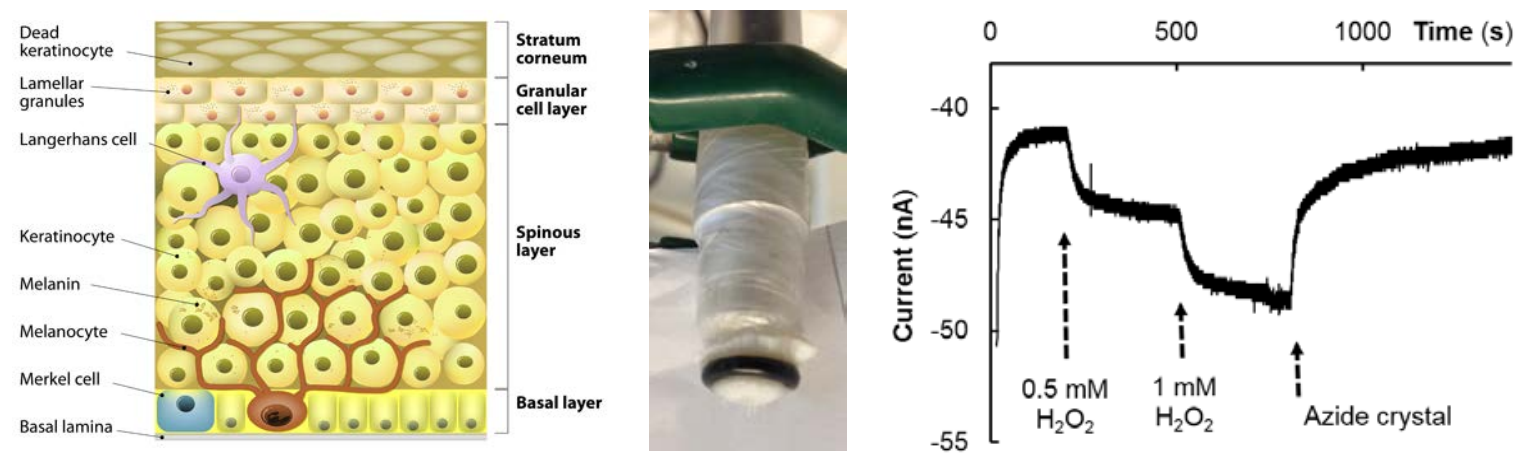

Figure: (left) Schematic presentation of layered structure of skin membrane consisting of stratum corneum (SC) and viable epidermis; (middle) a photo of oxygen electrode covered with skin membrane; (right) current of skin-covered oxygen electrode immersed in PBS, pH 7.4. Steady state baseline current (time interval: 100-200 s) is due to $\mathrm{O}_{2}$ dissolved in PBS. At 200 and $500 \mathrm{~s}_{2} \mathrm{O}_{2}$ is added resulting into 0.5 and $1 \mathrm{mM} \mathrm{H}_{2} \mathrm{O}_{2}$ concentration, respectively. The observed two current steps reflect activity of skin catalase. At $800 \mathrm{~s}$ the catalase inhibitor $\mathrm{NaN}_{3}$ was added to the solution, resulting in the current return to the baseline level. 


\section{Introduction}

Since the invention of biosensors in 1962 (Clark and Lyons 1962), the Clark oxygen electrode has been used to construct biosensors based on various enzymes, cells, and tissues (Turner et al. 1987). In most cases, the mentioned biological materials served as a recognition element providing specificity for the transduction function of the biosensor. In several cases the biosensor design has been used to address functions of the biomaterials itself, e.g., to monitor the activity of surface bound enzymes (Haberska et al. 2008; Ruzgas et al. 1995) or yeast cells attached at the electrode (Heiskanen et al. 2009; Spegel et al. 2007). Recently we proposed a skin covered electrode for studies of dynamics of transdermal penetration of biologically active compounds such as hydrogen peroxide, ascorbic acid, and quercetin (Gari et al. 2015; Rembiesa et al. 2015).

In this work we assessed the possibility of covering an oxygen electrode with excised pig skin membranes for investigation of native catalase function in skin. Catalase is one of the enzymes of the biological antioxidative system in skin (Pillai et al. 2005) where it removes hydrogen peroxide, $\mathrm{H}_{2} \mathrm{O}_{2}$, from skin by converting it to water and oxygen according to:

$$
2 \mathrm{H}_{2} \mathrm{O}_{2} \stackrel{\text { Catalase }}{\longrightarrow} 2 \mathrm{H}_{2} \mathrm{O}+\mathrm{O}_{2} \text { Eq. } 1
$$

$\mathrm{H}_{2} \mathrm{O}_{2}$ is one of the most stable forms of reactive oxygen species, ROS, which might be generated in skin by both exogenous and endogenous factors. Exogenous factors leading to elevated ROS in skin is, for example, exposure to UV irradiation, whereas chronic inflammation is an example of endogenous factor. Considering the general relevance of these examples, it is clear that monitoring the skin's ability to detoxify ROS is of great interest in a wide range of situations including healthcare settings and development of topical products such as sun screen lotions. To the best of our knowledge, this work represents the first study where it is demonstrated that the oxygen electrode can be utilized for electrochemical in-vitro monitoring of native catalase activity in skin. The proposed electrode design is a first step towards a simple and easy operated electrochemical tool to assess the function of native antioxidative system in skin.

As a background to help appreciate the results of this work, the skin structure is briefly described by the schematic drawing in Fig. 1a. It should be kept in mind that the structure of the skin is much more complicated, however, our simplified description is sufficient to understand the results of this work. Skin is one of the largest organ of the body and consists of several anatomically different layers as seen in Fig. 1a. The outermost layer is called the stratum corneum, SC, and is usually represented by the brick and mortar model, where the bricks represent dead cells (i.e. corneocytes), which are surrounded by a continuous lipid multilamellar matrix, i.e. the mortar (Michaels et al. 1975). Tape-stripping of the $\mathrm{SC}$ is often used to demonstrate that the barrier properties of the skin for transdermal penetration vanishes when the SC is stripped of (Valk and Maibach 1990). In particular, the continuous lipid lamellae matrix of SC represents a tough diffusional barrier towards transdermal penetration and thus ensures hydration homeostasis by minimizing the water loss from the body to the external environment (Iwai et al. 2012; Scheuplein and Blank 1971). Interestingly, the transdermal permeability of the skin barrier can be reversibly regulated by changing the degree of SC hydration (Björklund et al. 2010; Björklund et al. 2013). This example emphasizes the fact that the skin is a responding membrane, i.e., the membrane can change its properties following the changes of external biophysical factors (Björklund et al. 2010). Regulation of transdermal permeability as well as monitoring of enzyme activity in skin are, thus, of broad medical and industrial interest.

The results described in this work provide a number of important conclusions. Firstly, we demonstrate that the activity of native catalase 
in skin is considerable in freshly excised pig skin membranes and that it is possible to switch-off the catalase activity in skin by an enzyme inhibitor. Secondly, the catalase activity is high enough to detoxify transdermal penetration of $\mathrm{H}_{2} \mathrm{O}_{2}$ from a relatively concentrated solution of $\mathrm{H}_{2} \mathrm{O}_{2}$. Thirdly, from tape-stripping experiments we conclude that the catalase is primarily located in the viable epidermis and that the $\mathrm{H}_{2} \mathrm{O}_{2}$ penetration is significantly enhanced upon successive stripping of SC. Fourthly, the activity of native catalase is severely suppressed by changing the $\mathrm{pH}$ from neutral to acidic solution in contact with the SC barrier.

Taken together, several of the findings in the present study are important to consider when developing topical formulations. For example, when the skin becomes exposed to UV irradiation, i.e., the conditions when ROS and $\mathrm{H}_{2} \mathrm{O}_{2}$ production might be elevated and functioning of catalase in skin should not be compromised. Our experiments also indicate that some penetration enhancers, i.e., components of topical formulations, can interfere with catalase reaction in skin.

\section{Material and methods}

\section{Material}

Hydrogen peroxide $(30 \%, 10.3 \mathrm{M})$, phosphate buffer saline (PBS, pH 7.4) in tablets, sodium citrate and sodium chloride for preparation of citrate buffer saline (CBS, pH 4.0 consisting of $10 \mathrm{mM}$ sodium citrate and $150 \mathrm{mM} \mathrm{NaCl}$ ), thymol, and azide were purchased from Sigma Aldrich. Oxygen electrode, constructed using 5 $\mu \mathrm{m}$ thick Teflon membrane from DuPont Fluoropolymers (Detroit, MI, USA), $250 \mu \mathrm{m} \mathrm{Pt}$ melted in glass and internal $\mathrm{Ag} / \mathrm{AgCl}$ reference, was purchased from UAB "OPTRONIKA", Vilnius, Lithuania. All solutions have been prepared by using deionised water purified by Milli-Q system (Merck Millipore, Billerica, USA) with resistivity of $18.2 \Omega \mathrm{cm}$.

\section{Preparation of skin membranes}

Fresh pig ears were obtained from a local abattoir and stored at $-80^{\circ} \mathrm{C}$ if not immediately used. To prepare skin membranes, fresh or defrosted pig ears were rinse with cold water and cut into strips with a scalpel. Outer part (stratum corneum side) of the strip was shaved and approximately $500 \mu \mathrm{m}$ thick skin membranes were sliced with a dermatome. The resulting skin stripes were punched out to make circular membranes with $16 \mathrm{~mm}$ in diameter. If not immediately mounted on the oxygen electrode, the membranes were kept in the fridge $\left(+4^{\circ} \mathrm{C}\right)$ on a filter paper soaked with PBS. Skin membranes, prepared as described, were used within two weeks.

\section{Preparation of skin covered oxygen electrode (SCOE)}

The platinum cathode surface of the oxygen electrode was polished using alumina suspension ( $1 \mu \mathrm{m}$ alumina, Buehler, Lake Bluff, $\mathrm{IL}$ ) and rinsed with deionized water. The body of the electrode was filled with saturated $\mathrm{KCl}$ solution and covered with $5 \mu \mathrm{m}$ Teflon membrane. This constituted the Clark type oxygen electrode. This electrode was then covered with the skin membrane as shown in Fig. $1 b$.

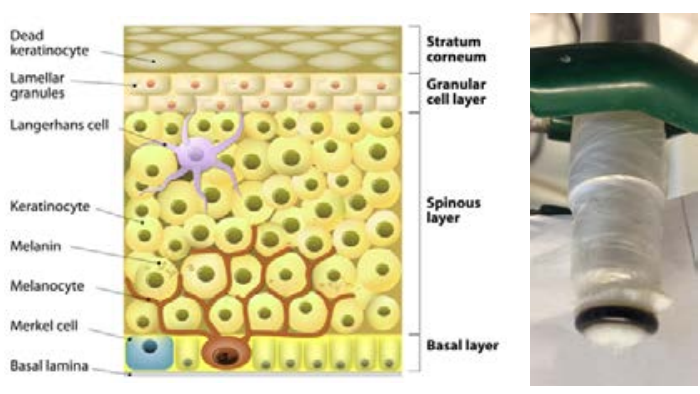

Fig. 1. (left) Schematic presentation of layered structure of skin membrane consisting of stratum corneum (SC) and viable epidermis (Creative Commons (Attribution 3.0) from freedesignfile.com) and (right) a photo of oxygen electrode covered with skin membrane.

\section{Amperometric monitoring of catalase activity in skin}

The skin membrane covered oxygen electrode (Fig. 1b) was dipped into an electrochemical cell filled with $20 \mathrm{~mL}$ PBS (pH 7.4) or CBS (pH 4.0) buffer. Electrochemical measurements were performed using a CompactStat 
potentiostat from IVIUM Technologies (Eindhoven, The Netherlands). The oxygen electrode was connected to the potentiostat in two electrode configuration by applying $-0.7 \mathrm{~V}$ vs $\mathrm{Ag} / \mathrm{AgCl} / \mathrm{KClsat}$ on Pt cathode of the oxygen electrode. After the baseline current was stabilized, a defined amount of $\mathrm{H}_{2} \mathrm{O}_{2}$ was pipetted into the electrochemical cell, which increased the reduction current of the oxygen electrode. In all cases, the solution was continuously mixed with a magnetic stirrer and all measurements were conducted at room temperature $\left(+21^{\circ} \mathrm{C}\right)$.

\section{Results and discussion}

To access the activity of native catalase in skin we employed split-thickness membranes (approx. $500 \mu \mathrm{m}$ thickness) and mounted them on the tip of the Clark-type oxygen electrode resulting in the skin covered oxygen electrode, SCOE, as shown in Fig. 1b. Upon immersion of the SCOE in a solution of PBS a stable baseline current is generated (Fig. 2, time interval 100$200 \mathrm{~s})$. The baseline current is related to the $\mathrm{O}_{2}$ diffusion from the PBS solution, across the skin membrane, to the oxygen electrode and is to a great extent determined by the $\mathrm{O}_{2}$ concentration of the PBS solution. This was confirmed by reducing the $\mathrm{O}_{2}$ concentration by passing $\mathrm{N}_{2}$ gas through the PBS solution, which resulted in that the baseline current approached zero (data not shown). As can be seen from Fig. 2a, stepwise addition of $\mathrm{H}_{2} \mathrm{O}_{2}$ into PBS results in an increase of the electrode current due to the increase of $\mathrm{O}_{2}$ at the electrode surface. The increase of $\mathrm{O}_{2}$ is caused by $\mathrm{H}_{2} \mathrm{O}_{2}$ diffusion into skin where $\mathrm{O}_{2}$ is generated due to the catalase reaction specified in Eq. 1. To confirm that the native catalase was responsible for the generation of $\mathrm{O}_{2}$ from the added $\mathrm{H}_{2} \mathrm{O}_{2}$, a few crystals of the catalase inhibitor, sodium azide $\left(\mathrm{NaN}_{3}\right)$, were added into the PBS at time $800 \mathrm{~s}$ (Fig. 2). It is clear that the $\mathrm{NaN}_{3}$ addition resulted in that the electrode current returned to the baseline level (Fig. 2, time interval 800-1400 s), which implies total inhibition of the native catalase activity. Taken together, the data in Fig. 2 demonstrate that the activity of native catalase in skin can be studied by the proposed SCOE setup (Fig. 1b). Below we illustrate the versatility of this setup by a few key experiments and discuss how the SCOE can be utilized to address important questions related to skin healthcare and cosmetic applications.

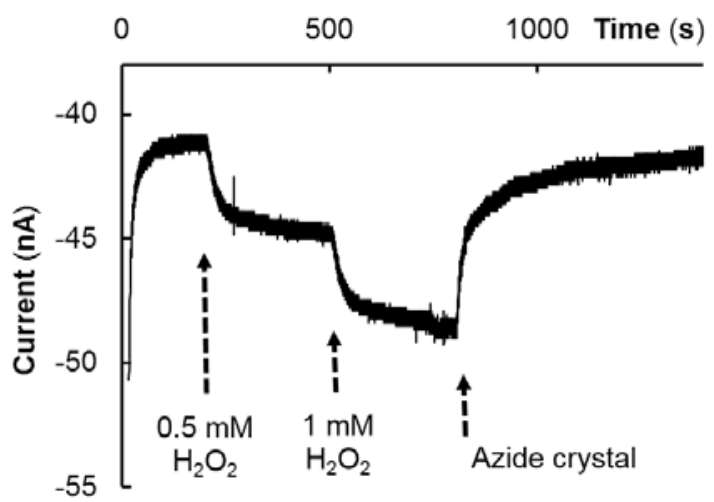

Fig.2. Current of skin-covered oxygen electrode immersed in PBS, $\mathrm{pH}$ 7.4. Steady state baseline current (time interval: 100-200 s) is due to $\mathrm{O}_{2}$ dissolved in PBS. At 200 and $500 \mathrm{~s} \mathrm{H}_{2} \mathrm{O}_{2}$ is added resulting into 0.5 and $1 \mathrm{mM}$ $\mathrm{H}_{2} \mathrm{O}_{2}$ concentration, respectively. The observed two current steps reflect activity of skin catalase as specified by Eq. 1. At $800 \mathrm{~s}$ the catalase inhibitor $\mathrm{NaN}_{3}$ was added to the solution, resulting in that the current returned to the baseline level.

The SC is one of the toughest biological barriers, which protects the body from the external environment as well as from dehydration due to minimal transepidermal water loss. It is well established that the SC is the diffusion limiting barrier in transdermal drug delivery (Scheuplein and Blank 1971). Permeability of SC can be increased by skin hydration (Björklund et al. 2010; Björklund et al. 2013), exploiting penetration enhances (Pham et al. 2016; Williams and Barry 2004) or simply removing this barrier by tape stripping (Valk and Maibach 1990). It has been shown that approximately one layer of corneocytes/keratinocytes is removed per single tape stripping application, but it should be noted that the cells may originate from various depths because of furrows in the skin (Molen et al. 1997). Nevertheless, repeated stripping of the SC results in a thinner and more defective skin barrier and after about 20 repetitions of this procedure SC usually 
contains regions where the barrier is completely removed (Molen et al. 1997; Valk and Maibach 1990). To understand the barrier properties of $\mathrm{SC}$ with respect to $\mathrm{H}_{2} \mathrm{O}_{2}$ penetration we investigated the effect of tape stripping on the current of the SCOE. The experiments were done by repeating the following experimental cycle: (i) amperometric registration of the response of the SCOE to 0.5 $\mathrm{mM} \mathrm{H} \mathrm{H}_{2} \mathrm{O}_{2}$, and (ii) tape stripping without removing the skin membrane from the electrode. The electrode response to $\mathrm{H}_{2} \mathrm{O}_{2}$ addition after different number of tape stripping of skin is demonstrated in Fig. 3.
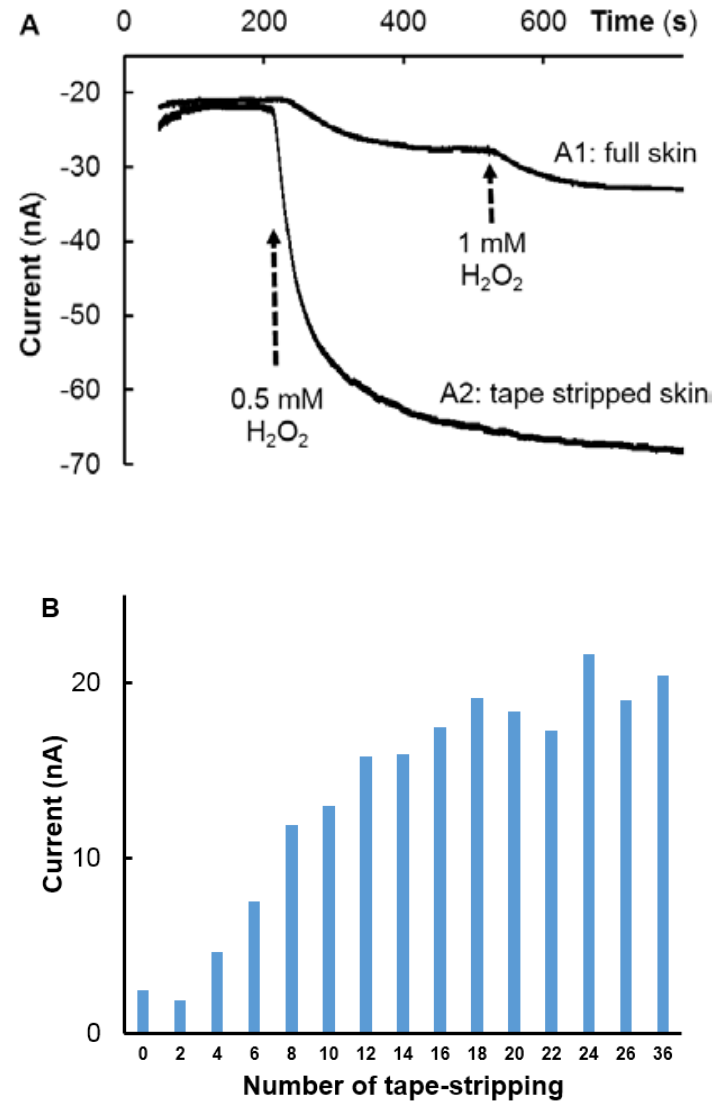

Fig.3. Amperometric response of skin-covered oxygen electrode (SCOE) to $\mathrm{H}_{2} \mathrm{O}_{2}$ before and after tape stripping. A: (A1) Amperometric response to two consecutive additions of $\mathrm{H}_{2} \mathrm{O}_{2}$ into PBS solution resulting into 0.5 and $1 \mathrm{mM} \mathrm{H} \mathrm{H}_{2} \mathrm{O}_{2}$ concentrations, respectively. (A2) Amperometric response of the electrode after 20-time tape stripping of skin membrane to the addition of $\mathrm{H}_{2} \mathrm{O}_{2}$ into PBS solution resulting into $0.5 \mathrm{mM} \mathrm{H}_{2} \mathrm{O}_{2}$ concentration. (B) The dependence of steady-state amperometric current on the tape-stripping number. The current is calculated as a difference between the electrode current in the presence of $\mathrm{H}_{2} \mathrm{O}_{2}$ minus the current in the absence of $\mathrm{H}_{2} \mathrm{O}_{2}$ in buffer solution (baseline current).

As can be seen in Fig. 3a, 20 applications of the tape strip method resulted in approximately an eigth-fold increase in the current response of the SCOE to $0.5 \mathrm{mM} \mathrm{H}_{2} \mathrm{O}_{2}$. The electrode response increases gradually with the number of tape strip applications, as demonstrated in Fig. 3B. As expected, after around 18-20 tape strip repetitions the current approaches a high and stable value, which corresponds to the minimal resistance of diffusional transport through the viable skin that is left once the skin's SC barrier is removed. Tape stripping results in approximately 10 times higher response of the electrode to $\mathrm{H}_{2} \mathrm{O}_{2}$. Several important conclusions can be drawn from the experiments conducted with SCOE in combination with the tape-stripping methodology. Firstly, SC acts as a substantial diffusion barrier even for such a small molecule as $\mathrm{H}_{2} \mathrm{O}_{2}$. The observation that the response of the electrode increases substantially with the number of tape stripping indicates that skin hosts a substantial activity of catalase. In other words, $\mathrm{H}_{2} \mathrm{O}_{2}$ penetrating from a $0.5-1 \mathrm{mM}$ $\mathrm{H}_{2} \mathrm{O}_{2}$ solution into the viable epidermis and underlying viable tissue will be converted to $\mathrm{O}_{2}$ and thus detoxified by native skin catalase. To understand the relevance of these conclusion it can be mentioned that 0.1-1 $\mathrm{mM} \mathrm{H}_{2} \mathrm{O}_{2}$ concentrations have been reported to promote wound healing or to be toxic to cells (Loo et al. 2011; Schreml et al. 2010). Peroxide containing antimicrobial creams and bleaching solutions might contain between $1 \%$ (300 mM) to $5 \%$ (1500 mM) of $\mathrm{H}_{2} \mathrm{O}_{2}$ (Chan and Maibach 2008). The experiments summarized in this paragraph demonstrate that the SCOE can be used to study barrier properties of skin for optimizing topical products containing $\mathrm{H}_{2} \mathrm{O}_{2}$.

In development of topical products, such as creams and lotions, certain $\mathrm{pH}$ values of the formulation are chosen. In some cases the choice is motivated with the aim to match the assumed skin $\mathrm{pH}$, or to increase the topical or transdermal delivery, or simply to increase the 
stability of the formulation for storage purposes. We have assessed the effect of $\mathrm{pH}$ on the activity of catalase in skin by recording the amperometric current response of the SCOE to $0.5 \mathrm{mM} \mathrm{H}_{2} \mathrm{O}_{2}$ at different $\mathrm{pH}$ ranging from 3.5 to 8.0. Typical experimental data are summarized in Fig. 4. As can be seen the response of the electrode is considerably reduced at lower $\mathrm{pH}$ implying that the activity of catalase in skin is noticeably suppressed at acidic $\mathrm{pH}$. The stepwise return to $\mathrm{pH} 7.4$ recovers some activity, however, the activity is lower as compared to the initial measurements. This observation can probably be explained by inactivation of catalase due to exposure to $\mathrm{H}_{2} \mathrm{O}_{2}$ at acidic $\mathrm{pH}$. However, this conclusion is restricted by the fact that the skin membrane may change between each experiment. The fact that the current response is decreased for the repeated measurement at the same $\mathrm{pH}$ implies irreversible changes of the skin membrane. However, it should be mentioned that the reduction of the electrode response at acidic $\mathrm{pH}$ shown in Fig. 4 is considerably more pronounced if compared to the loss of response after repeated measurements at $\mathrm{pH} 7.4$ (data not shown). The general conclusion is that the catalase activity in skin is considerably lower at acidic $\mathrm{pH}$. However, since the effect of $\mathrm{pH}$ on the rate of transdermal penetration of $\mathrm{H}_{2} \mathrm{O}_{2}$ across $\mathrm{SC}$ was not evaluated in these experiment this conclusion is associated with some uncertainty.

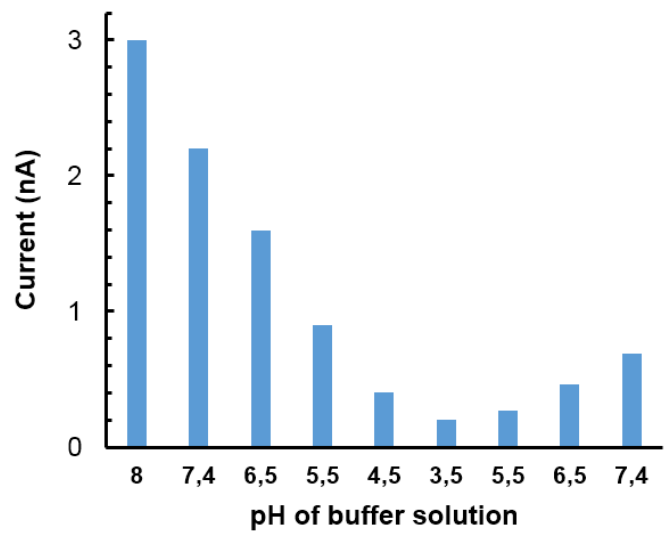

Fig.4. Amperometrically measured steady-state current of the skin covered oxygen electrode (SCOE) at $0.5 \mathrm{mM}$ of $\mathrm{H}_{2} \mathrm{O}_{2}$ in buffer solutions of different $\mathrm{pH}$. Phosphate buffer saline with $\mathrm{pH} 8.0,7.4$ and 6.5 and or citrate buffer saline with $\mathrm{pH} 4.5$ and 3.5. The current is calculated as a difference between the electrode current in the presence of $\mathrm{H}_{2} \mathrm{O}_{2}$ minus the current in the absence of $\mathrm{H}_{2} \mathrm{O}_{2}$ in buffer solution (baseline current).

It should be kept in mind that the SCOE is an invitro system and that the relevance of the observed $\mathrm{pH}$ effect on skin catalase activity might be questionable for the in-vivo situation where the $\mathrm{pH}$ of healthy viable epidermis should be constant and close to 7.4. However, a local reduction of the $\mathrm{pH}$ may be possible in cases of metabolic disturbances or diseases, including acute or chronic inflammation and cancer (Kato et al. 2013).

From the in-vitro measurements, summarized above, it is clear that acidic $\mathrm{pH}$ of topical formulations might reduce the activity of skin catalase, i.e. suppress the function of biological antioxidative system. This conclusion should have a significant interest and relevance in case when a formulation with low $\mathrm{pH}$ is applied on skin parts possessing elevated ROS production, e.g., in vitiligo disease (Schallreuter et al. 2001). This discussion about the function of catalase in skin leads us to a very complex and medically relevant area addressing interaction of formulations with the biological antioxidative system in skin. The potential use of the skincovered electrode setup to address some relevant questions in this context is demonstrated below.

Topical formulations are mixtures of chemical compounds with different functions, such as moisturizer, emollient, thickener, etc. The compounds that increase the rate of transdermal delivery are called penetration enhancers. Ideally they are supposed to be biologically "inert", i.e., not bioactive, and only act to change the physicochemical permeability properties of the skin barrier. By using skin-covered electrode for measurements of skin catalase activity we looked at the effect of the well know penetration enhance thymol (Pham et al. 2016). The chemical structure of thymol and the amperometric response of the skin- 
covered electrode to thymol, in the presence of $\mathrm{H}_{2} \mathrm{O}_{2}$, are presented in Fig. 5 .

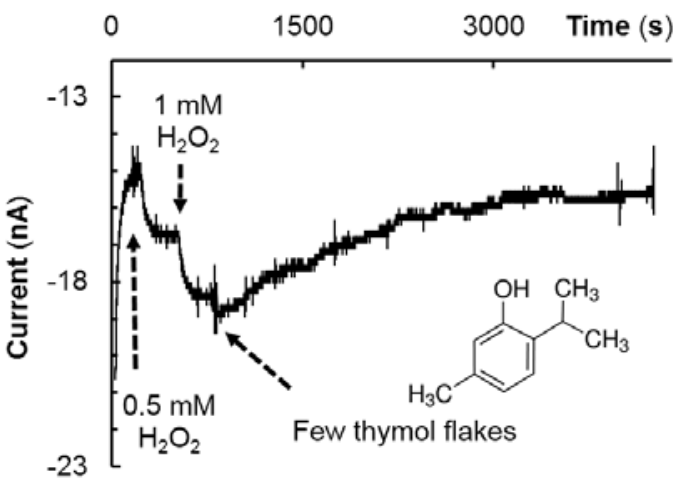

Fig. 5. Amperometric response of skin-covered electrode to two consecutive addition of $\mathrm{H}_{2} \mathrm{O}_{2}$ resulting in 0.5 and 1 $\mathrm{mM} \mathrm{H}_{2} \mathrm{O}_{2}$ concentration in PBS. Thymol was dropped into the electrochemical cells in amount of few flakes (thymol is sparingly soluble in water solution).

As can be seen from Fig. 5, two consecutive additions of $\mathrm{H}_{2} \mathrm{O}_{2}$ into PBS results into two similar amperometric current responses of the SCOE. The results indicate that catalase in skin is active and generates $\mathrm{O}_{2}$ from the $\mathrm{H}_{2} \mathrm{O}_{2}$, which penetrates through SC. At time $1000 \mathrm{~s}$ the thymol is added into the reaction mixture, resulting in a decrease of the electrode current. This is not what should be expected for the compounds, which is an extremely potent penetration enhancer (Pham et al. 2016). In other words, it is expected that thymol should increase the $\mathrm{SC}$ permeability and thus increase the $\mathrm{H}_{2} \mathrm{O}_{2}$ flux, leading to the increased electrode current. Nevertheless, even though the experimental results presented in Fig. 5 contradict the expected outcome, the results can sill be explained. As seen from the chemical structure of thymol, it contains a phenolic group. Phenolic structures can be oxidized by $\mathrm{H}_{2} \mathrm{O}_{2}$ catalyzed by catalases and peroxidases (Dunford 2010). In this case, the catalase will not generate $\mathrm{O}_{2}$ at the same rate as the peroxidase reaction consumes $\mathrm{H}_{2} \mathrm{O}_{2}$. Taking this reaction into consideration, the amperometric response of the skin-covered electrode to thymol in the presence of $\mathrm{H}_{2} \mathrm{O}_{2}$ is logical. Concluding, the experiments with thymol demonstrate that some compounds present in topical formulations can interfere with the biological antioxidative system in skin.
Since the compounds present in formulations have been clinically tested, in the majority of the cases this is probably not a problem. However, in cases when $\mathrm{H}_{2} \mathrm{O}_{2}$ is generated in skin, e.g., at UV irradiation and in cases of acute or chronic skin inflammations, their effect on antioxidative system of skin might be questionable. Finally, it should be emphasized that the proposed setup, based on the skincovered oxygen electrode, can be useful not only to measure catalase activity in skin but also provides a tool to address more complex functions of biological antioxidative system in skin.

\section{Conclusions}

In this work we demonstrate that the skincovered oxygen electrode (SCOE) allows investigation of catalase activity in skin. The setup comprises a skin membrane placed on the Clark type oxygen electrode. The catalase activity and function in skin is monitored based on the current response of the oxygen electrode. The current is due to $\mathrm{H}_{2} \mathrm{O}_{2}$ penetration through $\mathrm{SC}$ and subsequent catalytic generation of $\mathrm{O}_{2}$. By tape-stripping the SC we demonstrate that skin contains a substantial amount of catalase, which is sufficient to detoxify $\mathrm{H}_{2} \mathrm{O}_{2}$ that reaches the viable epidermis after exposure of skin to 0.5-1 $\mathrm{mM} \mathrm{H}_{2} \mathrm{O}_{2}$. Our results also indicate that catalase activity is reduced at acidic $\mathrm{pH}$ as compared to the activity at $\mathrm{pH}$ 7.4. Some reservation must be made since the effect of $\mathrm{pH}$, solely, on catalase activity is difficult to separate from the $\mathrm{pH}$ dependence of $\mathrm{H}_{2} \mathrm{O}_{2}$ permeability through SC. Nevertheless, we argue that the observed $\mathrm{pH}$ dependence might be relevant to in-vivo situations when local $\mathrm{pH}$ is reduced and ROS generation is elevated, e.g., by disease in skin. The experiments with penetration enhancer, thymol, shows that the SCOE can also be exploited to study broad spectrum of questions relevant to understand functioning of biological antioxidative system in skin. 


\section{Acknowledgements}

Financial support from The Knowledge Foundation (SB, JE), The Swedish Research Council (TR) and The Gustaf Th. Ohlsson Foundation (JE and TR) are greatly acknowledged. SN thanks ERASMUS program for scholarship.

\section{REFERENCES}

Björklund, S., Engblom, J., Thuresson, K., Sparr, E., 2010. A water gradient can be used to regulate drug transport across skin. J. Controlled Release 143, 191-200.

Björklund, S., Ruzgas, T., Nowacka, A., Dahi, I., Topgaard, D., Sparr, E., Engblom, J., 2013. Skin membrane electrical impedance properties under the influence of a varying water gradient. Biophys J. 104(12), 2639-2650.

Chan, H.P., Maibach, H.I., 2008. Hydrogen peroxide, bleaching, and skin: an overview. Cutaneous \& Ocular Toxicol. 27, 307-309.

Clark, L.C.J., Lyons, C., 1962. Electrode systems for continuous monitoring in cardiovascular surgery. Ann. NY Acad. Sci. 102, 29-45.

Dunford, H.B., 2010. Peroxidases and Catalases: Biochemistry, Biophysics, Biotechnology and Physiology, 2nd ed. WileyBlackwell, New Jersey.

Gari, H., Rembiesa, J., Masilionis, I., Vreva, N., Svensson, B., Sund, T., Hansson, H., Morén, A.K., Sjöö, M., Wahlgren, M., Engblom, J., Ruzgas, T., 2015. Amperometric in vitro monitoring of penetration through skin membrane. Electroanalysis 27, 111 - 117.

Haberska, K., Svensson, O., Shleev, S., Lindh, L., Arnebrant, T., Ruzgas, T., 2008. Activity of lactoperoxidase when adsorbed on protein layers. Talanta 76(5), 1159-1164.

Heiskanen, A., Spégel, C., Kostesha, N., Lindahl, S., Ruzgas, T., Emnéus, J., 2009. Mediatorassisted simultaneous probing of cytosolic and mitochondrial redox activity in living cells. Anal. Biochem. 384, 11-19.

Iwai, I., Han, H., Hollander, L.d., Svensson, S., Öfverstedt, L.-G., Anwar, J., Brewer, J., Bloksgaard, M., Laloeuf, A., Nosek, D., Masich, S., Bagatolli, L.A., Skoglund, U., Norlén, L.,
2012. The Human Skin Barrier Is Organized as Stacked Bilayers of Fully Extended Ceramides with Cholesterol Molecules Associated with the Ceramide Sphingoid Moiety. J. Invest. Dermatol. 132, 2215-2225.

Kato, Y., Ozawa, S., Miyamoto, C., Maehata, Y., Suzuki, A., Maeda, T., Baba, Y., 2013. Acidic extracellular microenvironment and cancer. Cancer Cell International 13(89), 1-8.

Loo, A.E.K., Ho, R., Halliwell, B., 2011. Mechanism of hydrogen peroxide-induced keratinocyte migration in a scratch-wound model. Free Rad. Biol. Med. 51, 884-892.

Michaels, A.S., Chandrasekaran, S.K., Shaw, J.E., 1975. Drug permeation through human skin. Theory and in vitro experimental measurement. AIChE J. 21(5), 985-996.

Molen, R.G.v.d., Spies, F., Noordende, J.M.v.t., Boelsma, E., Mommaas, A.M., Koerten, H.K., 1997. Tape stripping of human stratum corneum yields cell layers that originate from various depths because of furrows in the skin. Arch. Derm. Res. 289(9), 514-518.

Pham, Q.D., Björklund, S., Engblom, J., Topgaard, D., Sparr, E., 2016. Chemical penetration enhancers in stratum corneum Relation between molecular effects and barrier function. J. Controlled Release 232, 175-187.

Pillai, S., Oresajo, C., Hayward, J., 2005. Ultraviolet radiation and skin aging: roles of reactive oxygen species, inflammation and protease activation, and strategies for prevention of inflammation-induced matrix degradation - a review. Int. J. Cosmetic Sci. 27, 17-34.

Rembiesa, J., Gari, H., Engblom, J., Ruzgas, T., 2015. Amperometric monitoring of quercetin permeation through skin membranes. Int. J. Pharmaceutics 496, 636-643.

Ruzgas, T., Gorton, L., Emnéus, J., Marko-Varga, G., 1995. Kinetic models of horseradish peroxidase action on a graphite electrode. J. Electroanal. Chem. 391, 41-49.

Schallreuter, K.U., Moore, J., Wood, J.M., Beazley, W.D., Peters, E.M.J., Marles, L.K., Behrens-Williams, S.C., Dummer, R., Blau, N., ThoÈny, B., 2001. Epidermal $\mathrm{H} 2 \mathrm{O} 2$ Accumulation Alters Tetrahydrobiopterin 
(6BH4) Recycling in Vitiligo: Identi ${ }^{\circledR}$ cation of a General Mechanism in Regulation of All 6BH4Dependent Processes? J. Invest. Dermatol. 116(1), 167-174.

Scheuplein, R.J., Blank, I.H., 1971. Permeability of the skin. Physiol. Rev. 51(4), 702-747.

Schreml, S., Landthaler, M., Schaferling, M., Babilas, P., 2010. A new star on the H2O2rizon of wound healing? Exp. dermatol. 20, 229-231.

Spegel, C.F., Heiskanen, A.R., Kostesha, N., Johanson, T.H., Gorwa-Grauslund, M.-F., Koudelka-Hep, M., Emneus, J., Ruzgas, T., 2007. Amperometric Response from the Glycolytic versus the Pentose Phosphate Pathway in Saccharomyces cerevisiae Cells. Analytical Chemistry (Washington, DC, United States) 79(23), 8919-8926.

Turner, A., Karube, I., Wilson, G.S., 1987. Biosensors: Fundamentals and Applications. Oxford University Press, Oxford.

Valk, P.G.v.d., Maibach, H.I., 1990. A functional study of the skin barrier to evaporative water loss by means of repeated cellophane-tape stripping. Clin. Exp. Dermatol. 15(3), 180-182.

Williams, A.C., Barry, B.W., 2004. Penetration enhancers. Adv. Drug Deliv. Rev. 56(5), 603618. 